\title{
Molecular Dynamics Simulation of the Shear Viscosity of Molten Alkali Halides
}

\author{
Nuno Galamba, ${ }^{\dagger}$ Carlos A. Nieto de Castro, ${ }^{\dagger}$ and James F. Ely ${ }^{*, *}$ \\ Departamento de Quimica e Bioquimica e Centro de Ciencias Moleculares e Materiais, Faculdade de Ciencias \\ da Universidade de Lisboa, 1749-016 Lisboa, Portugal, and Chemical Engineering Department, \\ Colorado School of Mines, Golden, Colorado 80401-1887
}

Received: July 30, 2003; In Final Form: December 13, 2003

\begin{abstract}
Equilibrium molecular dynamics (EMD) Green-Kubo simulations of the shear viscosity coefficient of molten sodium chloride and potassium chloride are reported in the microcanonical ensemble $(N, V, E)$. The potential model used is the Born-Mayer-Huggins-Tosi-Fumi interionic potential, and several state points have been investigated. The form of the stress tensor elements for ionic fluids whose electrostatic interactions are calculated through the Ewald method is discussed in detail. The results show that the agreement between the simulation and the available experimental data is satisfactory, and the viscosity is overpredicted (generally to within $10 \%-15 \%)$.
\end{abstract}

\section{Introduction}

Molten salts have been studied for many years; however, renewed interest in them has increased, because of their use as electrolytes in high-temperature carbonate fuel cells. Under those high-temperature conditions, it is extremely difficult, if not impossible, to accurately measure transport properties (such as the viscosity and thermal conductivity) and data are very limited. A premise of this work is that, because of the experimental problems encountered at high temperature, it may be possible to simulate those properties more accurately than they can be measured. Thus, in this investigation, we have explored the accuracy with which the shear viscosity of "simple" ionic systems can be computed through molecular dynamics simulations, with the ultimate goal to extend these simulations to more complex and/or industrially relevant systems.

In this work, we have chosen the alkali halides as the subject of our investigation. Molten alkali halides are among the simplest ionic fluids; they are composed only of univalent monatomic ions, a fact which has long motivated the application of simulation methods to investigate their properties. We note that, even though simple molten salts have been the topic of computer simulation for many years, very few investigations of the transport properties of these materials have been reported in the literature. Noteworthy (but not inclusive) examples of these previous simulations include both equilibrium molecular dynamics (EMD) ${ }^{1-3}$ and Monte Carlo ${ }^{4,5}$ studies. Generally, these studies have only reported the thermodynamic state, the microscopic structure, and the self-diffusion coefficient.

Of the different interionic potentials that have been proposed for the simulation of molten alkali halides, the Born-MayerHuggins model, ${ }^{6-9}$ with parameters calculated by Tosi and Fumi $^{10,11}$ (BMHTF), has been preferred in most of the studies reported in the literature. Even though this model is based on the rigid ion approximation (thus neglecting the ion-induced dipole (polarization) type of interactions) and uses parameters obtained from solid-state data only, it reproduces the thermo-

* Author to whom correspondence should be addressed. E-mail: jely@ mines.edu.

University of Lisbon

$\div$ Colorado School of Mines. dynamic and structural properties of molten alkali halides reasonably well. For the case of the self-diffusion coefficients, the results are satisfactory, but the small set of experimental self-diffusion data available is a limitation in making conclusions about the accuracy of the model.

Despite possible improvements of the BMHTF interionic potential through the inclusion of polarization effects (e.g. shell model interionic potentials), ${ }^{12-14}$ the model's capability to reproduce the thermal conductivity or the shear viscosity coefficients is relatively unexplored. This article discusses the computation of the shear viscosity of molten $\mathrm{NaCl}$ and $\mathrm{KCl}$, using EMD simulations in conjunction with the Green-Kubo method for different temperatures; the volume at each temperature is calculated from experimental density data. ${ }^{15}$

Simulated molten alkali halide shear viscosities have been reported previously, but only at a single state point. The temperature and/or density dependence has never been the primary focus of those studies, nor has the microcanonical ensemble been used. In particular, Ciccotti et al. ${ }^{16}$ calculated the shear viscosity of six molten alkali halides ( $\mathrm{LiF}, \mathrm{NaCl}, \mathrm{NaI}$, $\mathrm{KI}, \mathrm{RbCl}$, and $\mathrm{RbI}$ ), using a nonequilibrium molecular dynamics (NEMD) method. The reported thermodynamic states corresponded to a single temperature and density close to the triple points of each of the salts studied. The incorporation of a polarization contribution to the interionic forces was investigated by Jacucci et al. ${ }^{14}$ for the viscosity simulation of one molten salt (KI). The polarization model led to a decrease in the shear viscosity and an increase in the difference between the simulations and available experimental data. An EMD-NVT study of the viscosity of molten $\mathrm{NaCl}$ and $\mathrm{KCl}$ was performed by Sindzingre and Gillan. ${ }^{17}$ However, the primary objective of that study was the thermal conductivity of solid $\mathrm{NaCl}$ and $\mathrm{KCl}$, and, again, only one point is reported for the viscosity of molten $\mathrm{NaCl}$ and $\mathrm{KCl}$. More recently, NEMD simulations of the shear viscosity of $\mathrm{NaCl}$ were reported by Delhommelle and Petravic, ${ }^{18}$ although the primary focus of that work was on a comparison of the performance of kinetic and configurational temperature thermostats used in NEMD simulations. The state point reported in that article has been simulated in this work and is given for comparison purposes. The objective of this work is to investigate 
the capability of the BMHTF potential model to estimate transport coefficients that are more complex than the selfdiffusion coefficient at state points for which comparison with available experimental data is possible.

The paper is organized as follows: First, a description of the model and the parameters used in the simulations is given. The Green-Kubo method is discussed, and the form of the stress-tensor elements for the case of ionic systems for which the Ewald sum is used for handling the long-range interactions is considered in detail. The reason for this discussion is that, in applying the Ewald method to the evaluation of the stress tensor, there are two possible interpretations of how the reciprocalspace force should be used in the calculation. The correct interpretation is not discussed in standard simulation references, ${ }^{19-21}$ and we found it worthwhile to compare the two interpretations. The results obtained in this work are then given and compared with available experimental data and results of previous simulations. Finally, the conclusions of this study are given.

\section{The Green-Kubo Viscosity for Ionic Systems}

The thermal transport coefficients can be calculated from the Green-Kubo formulas through integration of the appropriate autocorrelation functions (ACFs), which, in turn, can be computed from EMD simulations. ${ }^{19-22}$ One of the problems in calculating the viscosity through this approach is the fact that, unlike self-diffusion, which is a single-particle phenomenon, the shear stress involves the entire system. Thus, it is not possible to improve the statistical precision of the calculation by averaging the results over the $N$ particles that compose the system. This, in addition to the possible system size dependence of the simulations, leads to rather long runs. Improvement of the statistical precision can be achieved by averaging over the three different off-diagonal elements of the stress tensor $\ddot{\boldsymbol{J}}$. For potential models for which the force has the form $\boldsymbol{F}_{i j}=F_{i j} \boldsymbol{r}_{i j} /$ $r_{i j}$, as it is for the case of the BMHTF model, $J_{a b}=J_{b a}$, which limits the average to three elements only. ${ }^{22}$ Further improvement of the statistics can be obtained using the method of Daivis and Evans, ${ }^{23}$ which takes advantage of full symmetry properties of the stress-tensor elements. This method allows the inclusion of the diagonal elements of the stress tensor on the calculation of the averaged stress autocorrelation function (ACF). However, in this study, we have not used this method, and the stress ACFs were obtained from the three distinct off-diagonal elements. Thus, the shear viscosity can be written as

$$
\eta=\frac{1}{3 V k T} \int_{0}^{\infty}\left\langle\sum_{x y} J_{x y}(t) J_{x y}(t)\right\rangle \mathrm{d} t
$$

where the stress-tensor element $J_{x y}$, with analogous expressions for the other components, is given by ${ }^{24}$

$$
J_{x y}=\sum_{i}^{N} m_{i} v_{i x} v_{i y}+\sum_{i}^{N-1} \sum_{j>i}^{N} r_{i j y} F_{i j x}
$$

For nonionic fluids, eqs 1 and 2 are used directly to obtain the shear viscosity of the fluid of interest by calculating and integrating the averaged stress correlation function. For an ionic fluid, these equations can strictly only be used in the computation of the short-range portion of the forces and for the real component of the Ewald-Coulomb forces that are needed to compute the potential component of the stress-tensor elements. Notice that, for this portion of the force, the distance between pairs of particles is calculated using the minimum image convention and radial truncation; thus, for instance, the force $F_{i j}$ corresponds to the force between the particle $i$ and the particle $j$ or its closest image within a sphere of radii $L / 2$ centered at $i$. For the case of the reciprocal-space force, the corresponding force between a "pair" of ions $i j$ is given by

$$
F_{i j x}^{\mathrm{F}}=\frac{2 Z_{i}}{L^{2}} \sum_{h \neq 0} Z_{j} \frac{h_{x}}{|\boldsymbol{h}|^{2}} \exp \left(-\frac{\pi^{2}|\boldsymbol{h}|^{2}}{\alpha^{2} L^{2}}\right) \sin \left(\frac{2 \pi}{L} \boldsymbol{h} \cdot \boldsymbol{r}_{i j}\right)
$$

where $\boldsymbol{r}_{i j}$ is the distance between the ions $i$ and $j$ within the central box. However, the force given by eq 3 is not just the force between the ions $i$ and $j$; rather, it is the force between $i$ and $j$ and all of its images in the infinite reciprocal-space lattice (which, in practice, is truncated using the condition $0<|\boldsymbol{h}|^{2} \leq$ $|\boldsymbol{h}|_{\max }^{2}$ for a cubic box). For a system with long-range forces, additional consideration must be given to interactions between particles more than one-half box length away. Thus eq 2, which, for a system with periodic boundary conditions ( $\mathrm{pbc}$ ), is written as

$$
J_{x y}=\sum_{i}^{N} m_{i} v_{i x} v_{i y}+\frac{1}{2} \sum_{i}^{N} \sum_{j}^{N} \sum_{n}^{\prime}\left(r_{i j y}-n_{y} L\right) F_{i j x}(\boldsymbol{r}-\boldsymbol{n L})
$$

can be truncated for values of $n=0, \pm 1$ for the case of shortrange forces, but more values of $\boldsymbol{n}$ should be taken into consideration for long-range forces, which are computed in the reciprocal space if the Ewald method is used. In eq 4, the prime symbol indicates the exclusion of the interaction of a particle with itself, $i \neq j$ for $n=0$.

Equation 4 shows that, if the direct product $F_{i j a}^{\mathrm{F}} r_{i j b}$ is calculated using the distance between the ions in the central box, this will not correspond to the distances between the pairs of ions considered within the reciprocal-space summation for the computation of the forces. This problem was first encountered by Vieillefosse and Hansen ${ }^{25}$ and Bernu and Vieillefosse, ${ }^{26}$ who have shown that, although the Green-Kubo relations for the transport properties of nonionic fluids could be applied to the case of the one-component plasma model, some care had to be taken in defining the microscopic currents, to avoid divergences.

The problem can be handled if the Ewald method is applied to the product $F_{i j a}^{\mathrm{F}} r_{i j b}$, rather than applying the method to just the force and then computing its product with the distance between the ions within the central MD box. ${ }^{27,28}$ Thus, for the case of the BMHTF model for a molten alkali halide, the stresstensor elements can be calculated by

$$
\begin{aligned}
& J_{a b}=\sum_{i}^{N} m_{i} v_{i a} v_{i b}+\sum_{i}^{N-1} \sum_{j>i}^{N} r_{i j, a} F_{i j, b}^{\mathrm{R}}+ \\
& \frac{1}{2 \pi L} \sum_{\boldsymbol{h} \neq 0} A(\boldsymbol{h}) B_{a b}\left\{\left[\sum_{i=1}^{N} Z_{i} \cos \left(\frac{2 \pi}{L} \boldsymbol{h} \cdot \boldsymbol{r}_{i}\right)\right]^{2}+\right. \\
& {\left.\left[\sum_{i=1}^{N} Z_{i} \sin \left(\frac{2 \pi}{L} \boldsymbol{h} \cdot \boldsymbol{r}_{i}\right)\right]^{2}\right\} }
\end{aligned}
$$

where

$$
A(\boldsymbol{h})=\frac{\exp \left[-\pi^{2}|\boldsymbol{h}|^{2} /\left(\alpha^{2} L^{2}\right)\right]}{|\boldsymbol{h}|^{2}}
$$

and, for the $a b$ component of the tensor $\ddot{\boldsymbol{B}}(\boldsymbol{h}),{ }^{28}$ 


$$
B_{a b}=\delta_{a b}-\frac{2\left|\boldsymbol{h}_{a}\right|\left|\boldsymbol{h}_{b}\right|}{|\boldsymbol{h}|^{2}}-\left(\frac{2 \pi}{L}\right)^{2} \frac{\left|\boldsymbol{h}_{a}\right|\left|\boldsymbol{h}_{b}\right|}{2 \alpha^{2}}
$$

In eq $5, F^{\mathrm{R}}$ includes the short-range forces and the real component of the Ewald-Coulomb forces, both computed in real space.

Figure 1 compares the dimensionless non-normalized stress ACF, calculated using eq 5 and the following (incorrect) expression:

$$
J_{x y}=\sum_{i}^{N} m_{i} v_{i x} v_{i y}+\sum_{i}^{N-1} \sum_{j>i}^{N} r_{i j y} F_{i j x}^{\mathrm{R}}+\sum_{i}^{N} r_{i y} F_{i x}^{\mathrm{F}}
$$

where the Fourier potential term of the stress-tensor element is incorrectly defined, because the positions considered are those only subject to pbc and not to the Ewald summation. Notice that the following transformation was used (using Newton's third law):

$$
\begin{aligned}
\frac{1}{2} \sum_{i}^{N} \sum_{j}^{N} \sum_{n} F_{i j}\left(\boldsymbol{r}_{i j}-\boldsymbol{n} L\right)\left(\boldsymbol{r}_{i j}-\boldsymbol{n L}\right) & \\
& \sum_{i}^{N} \sum_{\boldsymbol{n}} F_{i}\left(\boldsymbol{r}_{i j}-\boldsymbol{n} L\right)\left(\boldsymbol{r}_{i}-\boldsymbol{n} L / 2\right)
\end{aligned}
$$

In eq $8, F_{i}^{\mathrm{F}}$ is the Fourier component of the force on particle $i$ due to the interaction with all the particles in the reciprocalspace lattice. However, the position coordinate $r_{i y}$ is the $y$-coordinate of particle $i$ inside the central cell that does not replicate the image particles' positions in the reciprocal-space lattice. The net result is that a completely different form for the stress ACF is obtained by wrongly using the lattice sum given by eq 3 as the force between a single pair of ions within the MD box.

Although none of the points discussed here are new, we find it worthwhile to demonstrate the effect of using eq 8 , in terms of the computed stress ACF. Furthermore, the reasons behind the need to use eq 5 are not discussed in standard references. ${ }^{19-22}$ We also note that a similar problem is observed in the calculation of the virial pressure of ionic systems $;{ }^{29}$ however, that problem can be avoided using the relation between the pressure and Coulombic energy. ${ }^{30}$

\section{Model and Simulation Parameters}

The Born-Mayer-Huggins-Tosi-Fumi (BMHTF) potential model has been used in the simulations discussed here. This model has the following form:

$$
\phi_{i j}(r)=Z_{i} Z_{j} \frac{e^{2}}{r}+A_{i j} \exp \left[B\left(\sigma_{i}+\sigma_{j}-r\right)\right]-\frac{C_{i j}}{r^{6}}-\frac{D_{i j}}{r^{8}}
$$

where the first term represents the Coulomb interaction, and the second term represents the Born-Huggins exponential repulsion, with parameters obtained by Tosi and Fumi. ${ }^{10,11}$ The third and fourth terms respectively correspond to the dipoledipole and dipole-quadrupole dispersion energies, with parameters obtained by Mayer. ${ }^{8}$

The simulations have been performed in the microcanonical ensemble for a cubic sample composed of 216 ions using pbc and the minimum image convention. The positions at time zero were defined as those corresponding to the face-centered cubic lattice of solid $\mathrm{NaCl}$, with the cations occupying octahedral holes, and the zero-time velocities were defined randomly and

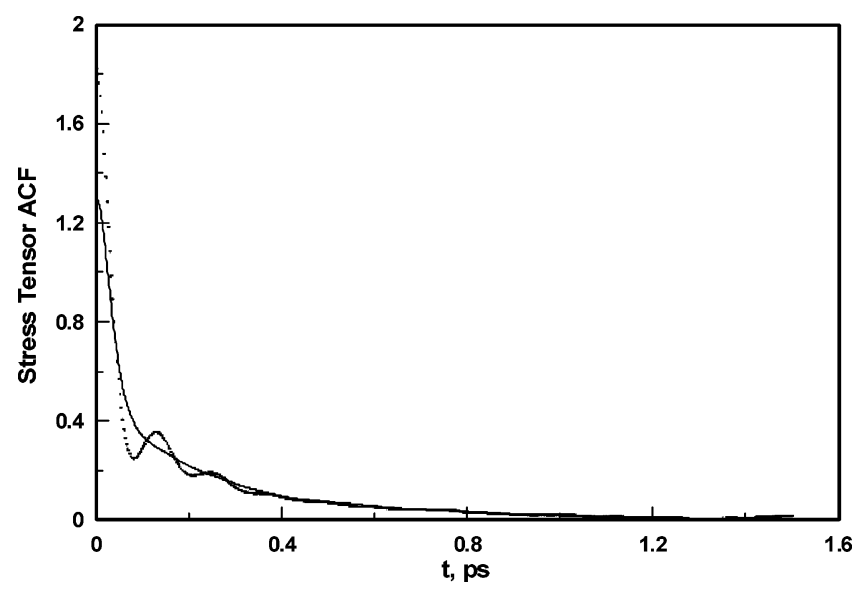

Figure 1. Comparison between the dimensionless stress autocorrelation function (ACF) calculated using eq 5 (solid line) and eq 8 (symbols). The correlation functions were calculated from a simulation of $\mathrm{NaCl}$ with $\rho=1.5420 \mathrm{~g} / \mathrm{cm}^{3}$ and $T^{\mathrm{sim}}=1091 \mathrm{~K}$.

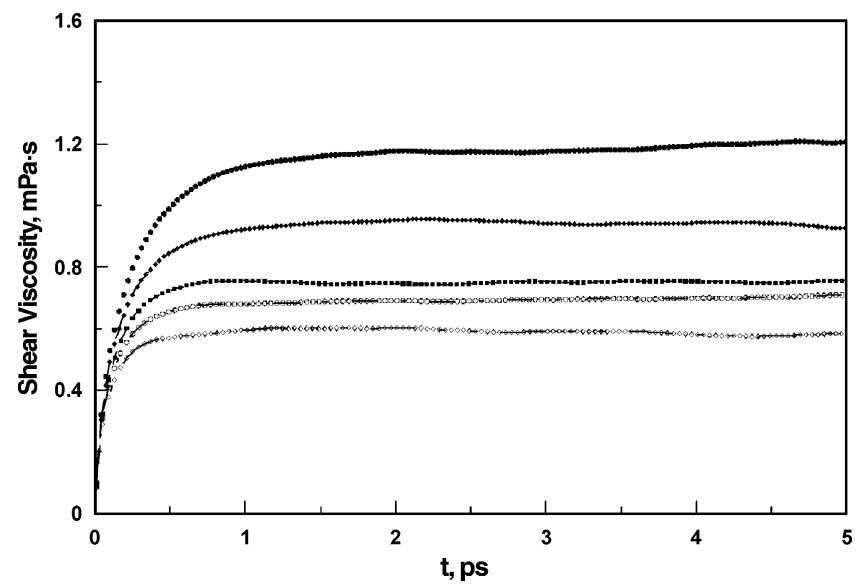

Figure 2. Integrated stress $\mathrm{ACFs}$ for $\mathrm{NaCl}$ for the different state points simulated. The simulation average temperatures, from top to bottom, are $T^{\text {sim }}=1091,1217,1339,1413$, and $1495 \mathrm{~K}$.

scaled to ensure a total linear momentum of zero. The Verlet "leapfrog" algorithm has been used to solve Newton's equations of motion, ${ }^{19}$ with a time step of $1 \times 10^{-15} \mathrm{~s}$, which ensures conservation of the total energy of the system. The system was equilibrated for $150 \mathrm{ps}$ at the desired temperature by scaling the velocities. The production stage was run for $5 \mathrm{~ns}$ for every point and the off-diagonal stress-tensor elements were accumulated at every time step. From the stress-tensor elements, the stress ACF was calculated and the shear viscosity coefficient obtained through numerical integration for a time window of 5 ps. The choice of the relatively small time step of $1 \mathrm{fs}$, in conjunction with the short-time spacing of the stress-tensor elements, generates negligible errors associated with the numerical integration of the stress ACF. For the simulations reported here, the value of the Ewald convergence parameter $\alpha$ was used ( $\alpha=5.6 / L$, where $L$ is the length of the side of the molecular dynamics box), with a truncation of the real component of the Ewald-Coulomb potential at $r_{c}=L / 2$ (thus using the same set of ion pairs as that for the short-range component of the potential energy and force), and the Fourier component of the force and potential energy was summed up to the vector $|\boldsymbol{h}|_{\max }^{2}=27$, with the $k$-space vector given by $\boldsymbol{k}=2 \pi \boldsymbol{h} / L$.

\section{Results and Comparisons}

Figure 2 shows the results of the integration of the stress $\mathrm{ACFs}$ at the different state points simulated for the case of $\mathrm{NaCl}$ 
TABLE 1: Simulation Results for $\mathrm{NaCl}^{a}$

\begin{tabular}{ccccccc}
\hline \multirow{2}{*}{$\begin{array}{c}T^{\text {equil }} \\
(\mathrm{K})^{b}\end{array}$} & $\begin{array}{c}T^{\text {sim }} \\
(\mathrm{K})\end{array}$ & $\begin{array}{c}\rho \\
\left(\mathrm{g} / \mathrm{cm}^{3}\right)\end{array}$ & $\begin{array}{c}P \\
(\mathrm{MPa})\end{array}$ & $\begin{array}{c}E \\
(\mathrm{~kJ} / \mathrm{mol})\end{array}$ & \multicolumn{2}{c}{ Viscosity $(\mathrm{mPa} \mathrm{s})$} \\
\hline 1100 & 1091 & 1.5420 & 151.7 & -691.4 & $1.18 \pm 0.01$ & 1.022 \\
1200 & 1217 & 1.4878 & 163.9 & -683.2 & $0.944 \pm 0.007$ & 0.811 \\
1300 & 1339 & 1.4335 & 165.8 & -675.3 & $0.750 \pm 0.004$ & 0.675 \\
1400 & 1413 & 1.3793 & 121.8 & -669.8 & $0.696 \pm 0.006$ & 0.614 \\
1500 & 1495 & 1.3250 & 91.04 & -663.8 & $0.590 \pm 0.009$ & 0.558
\end{tabular}

${ }^{a}$ The stress autocorrelation functions were evaluated from 5-ns productions runs $\left(5 \times 10^{6}\right.$ time origins $)$ and a time window of 5 ps. The experimental results are from Janz. ${ }^{15}{ }^{b}$ Equilibration temperature.

TABLE 2: Simulation Results for $\mathrm{KCl}^{a}$

\begin{tabular}{|c|c|c|c|c|c|c|}
\hline \multirow{2}{*}{$\begin{array}{l}T^{\text {equil }} \\
(\mathrm{K})^{b}\end{array}$} & \multirow{2}{*}{$\begin{array}{l}T^{\mathrm{sim}} \\
(\mathrm{K})\end{array}$} & \multirow{2}{*}{$\begin{array}{c}\rho \\
\left(\mathrm{g} / \mathrm{cm}^{3}\right)\end{array}$} & \multirow{2}{*}{$\begin{array}{c}P \\
(\mathrm{MPa})\end{array}$} & \multirow{2}{*}{$\begin{array}{c}E \\
(\mathrm{~kJ} / \mathrm{mol})\end{array}$} & \multicolumn{2}{|c|}{ Viscosity (mPa s) } \\
\hline & & & & & $\eta$ & $\eta^{\exp }$ \\
\hline 1050 & 1053 & 1.5236 & 240.3 & -626.7 & $1.259 \pm 0$ & 1.0 \\
\hline 1100 & 1133 & 1.4945 & 256.8 & -621.6 & $1.007 \pm 0.006$ & 0.881 \\
\hline 1200 & 1219 & 1.4362 & 213.5 & -615.4 & $0.878 \pm 0.001$ & 0.730 \\
\hline 1250 & 1231 & 1.4070 & 165.9 & -613.8 & $0.804 \pm 0.003$ & 0.713 \\
\hline 1300 & 1357 & 1.3779 & 214.9 & -606.6 & $0.654 \pm 0.005$ & 0.568 \\
\hline
\end{tabular}

${ }^{a}$ The stress autocorrelation functions were evaluated from 5-ns productions runs $\left(5 \times 10^{6}\right.$ time origins $)$ and a time window of $5 \mathrm{ps}$. The experimental results are from Janz. ${ }^{15}{ }^{b}$ Equilibration temperature.

TABLE 3: Comparison of Results Obtained in Different Simulation Studies of the Shear Viscosity of $\mathrm{NaCl}$ and $\mathrm{KCl}$

\begin{tabular}{|c|c|c|c|c|c|}
\hline salt & reference & $N_{\text {ions }}$ & $T(\mathrm{~K})$ & $\rho\left(\mathrm{g} / \mathrm{cm}^{3}\right)$ & $\eta(\mathrm{mPa} \mathrm{s})$ \\
\hline $\mathrm{NaCl}$ & Ciccotti et al. ${ }^{16}$ & 216 & 1262 & 1.495 & 0.87 \\
\hline $\mathrm{NaCl}$ & this work & 216 & 1217 & 1.488 & 0.94 \\
\hline $\mathrm{NaCl}$ & Sindzingre and Gillan ${ }^{17}$ & 216 & 1100 & 1.538 & 1.16 \\
\hline $\mathrm{NaCl}$ & this work & 216 & 1091 & 1.542 & 1.18 \\
\hline $\mathrm{NaCl}$ & Delhommelle and Petravic ${ }^{18}$ & 512 & 1500 & 1.5 & 0.791 \\
\hline $\mathrm{NaCl}$ & this work & 216 & 1526 & 1.5 & 0.765 \\
\hline $\mathrm{KCl}$ & Sindzingre and Gillan ${ }^{17}$ & 64 & 1096 & 1.495 & 1.24 \\
\hline $\mathrm{KCl}$ & this work & 216 & 1133 & 1.495 & 1.01 \\
\hline
\end{tabular}

(similar curves, not displayed here, were obtained for $\mathrm{KCl}$ ). The simulation results for the pressure, internal energy, and shear viscosity of $\mathrm{NaCl}$ and $\mathrm{KCl}$ are given in Tables 1 and 2. Because no thermostat was used in the simulations, the average temperatures for some points are very different from the input temperatures given in the first column of the tables. For the points where the average temperature is higher (lower) than the input temperature, a higher (lower) viscosity value is expected, because the experimental liquid densities at those points are lower (higher) than the values used in the simulations. The experimental data for the case of $\mathrm{NaCl}$ are slightly overpredicted; ${ }^{15}$ the error increases as the temperature increases. For the case of $\mathrm{KCl}$, the equation from which the viscosity has been calculated $^{15}$ is valid only for the temperature interval of $1111-$ $1142 \mathrm{~K}$ and extrapolated data have been used for the comparisons given in Table 2. Tables 1 and 2 show that the BHMTF rigid ion model overpredicts the shear viscosity for the two molten salts. As mentioned previously, the inclusion of a polarization model and its effect on the shear viscosity was investigated by Jacucci et al., ${ }^{14}$ and the results show a decrease in the simulated shear viscosity. That result indicates the possibility of obtaining even better agreement with the experimental data for these systems if polarization effects are taken into consideration.

Table 3 compares the values obtained in previous simulation studies with those obtained here. An additional point (see Table 3) has been simulated to compare these results with the recent simulations of $\mathrm{NaCl}$ through NEMD. ${ }^{18}$ The BMHTF model has been used in all the simulations whose results are given in Table 3. The value given in Table 3 from the work of Delhommelle and Petravic ${ }^{18}$ was obtained using the NEMD method with the iso-kinetic Gaussian thermostat. The values obtained by these authors, through NEMD (using a configurational temperature thermostat) and through EMD, were 0.783 and $0.782 \mathrm{mPa} \cdot \mathrm{s}$, respectively. Table 3 shows that the results obtained in the different studies are consistent with the results reported here.

\section{Conclusions}

Equilibrium molecular dynamics simulations of molten $\mathrm{NaCl}$ and $\mathrm{KCl}$ in the microcanonical ensemble have been performed in this work, to assess the degree of accuracy with which the shear viscosity of these systems can be computed through the rigid ion Born-Mayer-Huggins-Tosi-Fumi (BMHTF) model. The results obtained are in satisfactory agreement with the available experimental data, which are generally overpredicted within $10 \%-15 \%$.

Furthermore, a detailed discussion has been given on the form of the reciprocal-space portion of the stress-tensor elements for ionic systems for which the Ewald sum is used to handle the long-range forces. A comparison with previous simulations on the shear viscosity of molten alkali halides showed that the results obtained in this work are consistent with those of other authors.

Although the inclusion of polarization effects is expected to improve the results for the shear viscosity of molten alkali halides, further investigation of this point should give an account of the degree of improvement of the simulation data.

Acknowledgment. The authors would like to thank Dr. D. M. Heyes (University of Surrey) for a discussion on the concept of non-pairwise additive reciprocal-space forces and for noting the recent article by R. G. Winkler (ref 29). N.G. would like to acknowledge Fundacao para a Ciencia e Tecnologia for fellowship support (through the program PRAXIS XXI/BD/19792/ 99) and the Colorado School of Mines, where part of this work was developed, for the opportunity provided as a visiting researcher. J.F.E. was supported by the U.S. Department of Energy (through Grant No. DE-FG-ER41568).

\section{References and Notes}

(1) Lantelme, F.; Turq, P.; Quentrec, B.; Lewis, J. Mol. Phys. 1974, $28,1537$.

(2) Lewis, J. W. E.; Singer, K. J. Chem. Soc., Faraday Trans. 2 1975, $71,41$.

(3) Guissani, Y.; Guillot, B. J. Chem. Phys. 1994, 101, 490.

(4) Woodcock, L. V.; Singer, K. Trans. Faraday Soc. 1971, 67, 12

(5) Lewis, J. W. E.; Singer, K.; Woodcock, L. V. J. Chem. Soc., Faraday Trans. 2 1975, 71, 301.

(6) Born, M. V.; Mayer, J. E. Z. Phys. 1932, 75, 1.

(7) Huggins, M. L.; Mayer, J. E. J. Chem. Phys. 1933, 1, 643.

(8) Mayer, J. E. J. Chem. Phys. 1933, 1, 270.

(9) Huggins, M. L. J. Chem. Phys. 1937, 5.

(10) Fumi, F. G.; Tosi, M. P. J. Phys. Chem. Solids 1964, 25, 31.

(11) Tosi, M. P.; Fumi, F. G. J. Phys. Chem. Solids 1964, 25, 45.

(12) Dixon, M.; Sangster, M. J. L. J. Phys. C: Solid State Phys. 1975, 8 , L8.

(13) Dixon, M.; Sangster, M. J. L. J. Phys. C: Solid State Phys. 1976 9, L5.

(14) Jacucci, G.; McDonald, I. R.; Rahman, A. Phys. Rev. A 1976, 13, 1581

(15) Janz, G. J. NIST Properties of Molten Salts Database, 2.0 ed.; NIST SRD 27; NIST: Boulder, CO, 1992.

(16) Ciccotti, G.; Jacucci, G.; McDonald, I. R. Phys. Rev. A 1976, 13 426.

(17) Sindzingre, P.; Gillan, M. J. J. Phys.: Condens. Matter 1990, 2 , 7033.

(18) Delhommelle, J.; Petravic, J. J. Chem. Phys. 2003, 118, 2783.

(19) Allen, M. P.; Tildesley, D. J. Computer Simulation of Liquids; Oxford University Press: Oxford, U.K., 1987.

(20) Haile, J. M. Molecular Dynamics Simulation. Elementary Methods; Wiley: New York, 1992. 
(21) Frenkel, D.; Smit, B. Understanding Molecular Simulation. From Algorithms to Applications; Academic Press: New York, 2002.

(22) Rapaport, D. C. The Art of Molecular Dynamics Simulation; Cambridge University Press: New York, 1998.

(23) Daivis, P. J.; Evans, D. J. J. Chem. Phys. 1994, 100, 541.

(24) Zwanzig, R. Annu. Rev. Phys. Chem. 1965, 16, 67.
(25) Vieillefosse, P.; Hansen, J. P. Phys. Rev. A 1975, 12, 1106.

(26) Bernu, B.; Vieillefosse, P. Phys. Rev. A 1978, 18, 2345.

(27) Nose, S.; Klein, M. L. Mol. Phys. 1983, 50, 1055.

(28) Heyes, D. M. Phys. Rev. B: Condens. Matter 1994, 49, 755

(29) Winkler, R. G. J. Chem. Phys. 2002, 117, 2449.

(30) Sangster, M. J. L.; Dixon, M. Adv. Phys. 1976, 25, 247. 